\title{
SOLUTION OF A NONLINEAR PARTIAL DIFFERENTIAL EQUATION WITH INITIAL CONDITIONS
}

\author{
JAMES L. REID AND W. M. PRITCHARD
}

ABSTRACT. The exact solution $\phi$ of a particular nonlinear partial differential equation is obtained in terms of solution $\boldsymbol{u}$ of a related linear partial differential equation. It is noted that solution $\phi$ may be found subject to initial conditions if certain initial conditions can be determined for solution $u$. Two examples are solved explicitly.

R. T. Herbst [1] has pointed out that the ordinary nonlinear differential equation

$$
y^{\prime \prime}+p(x) y^{\prime}+k q(x) y=(1-l) y^{\prime 2} y^{-1}+\beta q(x) y^{1-l}
$$

has the solution

$$
y=[u+l \beta]^{k}, \quad k l=1, \beta=\mathrm{const},
$$

provided that $u$ satisfies the ordinary linear differential equation

$$
u^{\prime \prime}+p(x) u^{\prime}+q(x) u=0 .
$$

The purpose of this short note is to observe that (1) is readily generalized to the partial differential equation (6), below, in $n$ independent variables $x=\left(x_{1}, \cdots, x_{n}\right)$.

To obtain this generalization, define the operator

$$
L_{k}=\sum_{i, j=1}^{n} a_{i j}(x) \frac{\partial^{2}}{\partial x_{i} \partial x_{j}}+\sum_{i=1}^{n} b_{i}(x) \frac{\partial}{\partial x_{i}}+k c(x) ;
$$

substitute $\phi(x)$ defined by

$$
\phi(x)=[u(x)+\beta l]^{k}
$$

into the nonlinear differential equation

$$
L_{k} \phi=f(x, \phi, \partial \phi / \partial x),
$$

Received by the editors December 26, 1972 and, in revised form, March 12, 1973 and April 6, 1973.

AMS (MOS) subject classifications (1970). Primary 35C05; Secondary 35G10, $35 \mathrm{G} 25$.

Key words and phrases. Exact solution, nonlinear partial differential equation, linear nonhomogeneous partial differential equation, initial conditions.

(c) American Mathematical Society 1974 
where $f(x, \phi, \partial \phi / \partial x)$ represents the nonlinear terms to be determined; and, finally, make use of the assumption that $u(x)$ satisfies the linear equation

$$
L_{1} u=g(x),
$$

where $L_{1}$ is (4) with $k=1$. The calculation thus amounts to carrying out the operations indicated by (6), this procedure providing an identity for $f$. The details are straightforward and are omitted.

Thus the nonlinear partial differential equation

$$
L_{k}=(1-l) \phi^{-1} \sum_{i, j=1}^{n} a_{i j}(x) \frac{\partial \phi}{\partial x_{i}} \frac{\partial \phi}{\partial x_{j}}+[\beta c(x)+k g(x)] \phi^{1-l}
$$

is satisfied by (5), provided $u(x)$ satisfies the linear partial differential equation (7). Let this linear equation be called the base equation. The function $g(x)$ is arbitrarily prescribed; its presence in (7) extends the ordinary differential equation of Herbst.

If, in a given problem, the nonlinear term $\phi^{1-l}$ is present while $c(x) \equiv 0$, it is obvious that the base equation must be nonhomogeneous. When $\beta c(x) \not \equiv 0$, the base equation may be homogeneous if the product $\beta c(x)$ can be adjusted, as is always the case if $c$ is constant, to match a given coefficient of $\phi^{1-l}$. The arbitrariness of $\beta$ and $g(x)$ provides some flexibility in adapting (8) to a specified nonlinear equation.

An initial value problem can be posed for the solution $\phi(x)$ of the nonlinear equation (8) in terms of the solution $u(x)$ of the linear equation (7). Let $x_{0}$ denote initial values for any $m$ of the $n$ independent variables and let $z$ denote the remaining $n-m$ variables, such that

$$
\phi\left(z, x_{0}\right)=\phi_{0}(z)
$$

represents a specified function of the $x_{0}$ initial values and $z$. The function

$$
u\left(z, x_{0}\right)=u_{0}(z)
$$

is to be determined, and, from (5), it clearly must be

$$
u_{0}(z)=\left[\phi_{0}(z)\right]^{l}-\beta l,
$$

where $\phi_{0}(z) \neq 0$ if $l<0$. Therefore if $u(x)$ satisfies the linear equation (7) and the initial condition (11), then $\phi(x)$ satisfies the nonlinear equation (8) and the initial condition (9).

Similarly, if an initial condition on any of the first derivatives of $\phi(x)$ is specified then a corresponding derivative of $u(x)$ may be determined. For example, suppose that

$$
\partial \phi(x) /\left.\partial x_{i}\right|_{x_{i}=x_{i 0}}=F(z),
$$


where $F(z)$ is some function specified at $x_{i 0}$. It is thus required that

$$
\partial u(x) /\left.\partial x_{i}\right|_{x_{i}=x_{i 0}}=l\left[\phi\left(z, x_{i 0}\right)\right]^{l-1} F(z),
$$

where $\phi\left(z, x_{i 0}\right) \neq 0$ if $l<1$.

On the other hand, the specification of initial values for $u(x)$ and $\partial u(x) / \partial x_{i}$ imposes initial values on $\phi(x)$ and $\partial \phi(x) / \partial x_{i}$. Two explicit initial value problems are discussed below, after some special forms of (8) are noted.

For the special case $a_{i j}=0, i \neq j$, and $a_{i i} \equiv a_{i}$ constant, it is possible to put (8) in the form

$$
\begin{aligned}
\nabla_{a}^{2} \phi+\sum_{i=1}^{n} b_{i}(x) \frac{\partial \phi}{\partial x_{i}} & +k c(x) \phi \\
& =(1-l) \phi^{-1}\left(\nabla_{a} \phi\right)^{2}+[\beta c(x)+k g(x)] \phi^{1-l},
\end{aligned}
$$

where $\sqrt{ } a_{i} \partial / \partial x_{i}$ is the $i$ th component of a slightly generalized gradient operator $\nabla_{a}$ such that

$$
\nabla_{a}^{2} \phi=\sum_{i=1}^{n} a_{i} \frac{\partial^{2} \phi}{\partial x_{i}^{2}} \text { and }\left(\nabla_{a} \phi\right)^{2}=\sum_{i=1}^{n} a_{i}\left(\frac{\partial \phi}{\partial x_{i}}\right)^{2} .
$$

A nonlinear extension of the $n$-dimensional heat equation considered by Widder [2] follows from (14) in the form

$$
\nabla_{a}^{2} \phi-\partial \phi / \partial t=(1-l) \phi^{-1}\left(\nabla_{a} \phi\right)^{2}+g(x) \phi^{1-l},
$$

having a solution from the solution $u$ of the base equation

$$
\nabla_{a}^{2} u-\partial u / \partial t=g(x),
$$

where $t$ denotes a time variable. In this case, the coefficients $a_{i i}$ are all unity. The generalized gradient as defined above allows a nonlinear version of the equation studied by Lo [3] to take the same form as (15) but with $a_{i}=1, i=1, n-1$, say, and $a_{n}=\varepsilon$. A nonlinear Klein-Gordon equation would appear in this notation as

$$
\nabla_{a}^{2} \phi+k M^{2} \phi=(1-l) \phi^{-1}\left(\nabla_{a} \phi\right)^{2}+k g(x) \phi^{1-l}, \quad \beta \equiv 0,
$$

with $a_{i}=\sqrt{ }-1, i=1,3 ; a_{4}=1$; and $M=$ constant. A solution of (17) would follow from the nonhomogeneous Klein-Gordon equation

$$
\nabla_{a}^{2} u+M^{2} u=g(x) .
$$

The idea of devising a solution of a nonlinear partial differential equation from that of a related linear partial differential equation has been applied by Montroll ([4], [5]) to models of population growth and 
diffusion. He has considered, among others, nonlinear equations of the form

$$
D \nabla^{2} \phi-\partial \phi / \partial t=-D\left\{1-\left[G^{\prime}(\phi) / G(\phi)\right](\nabla \phi)^{2}\right\}+K G(\phi),
$$

where $D$ and $K$ are constants. Montroll solved this equation for $G(\phi)=$ $\phi(\theta-\phi) / \theta, \theta=$ constant, with several initial conditions. It is noted that (15) provides another type of nonlinear diffusion equation for which an exact solution is possible.

As an explicit example, consider the one-dimensional form of (15), i.e.,

$$
D \phi_{x x}-\phi_{t}=(1-l) \phi^{-1} D \phi_{x}^{2}+g(x) \phi^{1-l},
$$

where $x$ now denotes a single space variable defined in a closed interval $0 \leqq x \leqq L$. This one-dimensional equation is chosen for convenience to avoid undue complications. The problem is to solve (20) subject to the initial condition

$$
\phi(x, 0)=\phi_{0}(x)=0 .
$$

The condition that $u(x)$ must meet follows from (11) with $\beta=0$ :

$$
u(x, 0)=u_{0}(x)=\left[\phi_{0}(x)\right]^{l}=0, \quad l>0 .
$$

Thus an initial value problem is possible if $l$ is positive. A solution of the base equation (16) is known [6, p. 288] to be

$$
u(x, t)=\sum_{m=1}^{\infty}\left\{\int_{0}^{t} g_{m}(\tau) \exp \left[-D(m \pi / L)^{2}(t-\tau)\right] d \tau\right\} \sin (m \pi x / L),
$$

with

$$
\begin{aligned}
g(x, t) & =-\sum_{m=1}^{\infty} g_{m}(t) \sin (m \pi x / L), \\
g_{m}(t) & =\frac{2}{L} \int_{0}^{L} g(\xi, t) \sin (m \pi \xi / L) d \xi .
\end{aligned}
$$

Clearly, $u(x, 0)=0$ is possible from (23), and, hence, a solution of (20) and (21) is given by (23), the combination and (5). For this case, (20) is also satisfied for the boundary values

$$
\phi(0, t)=0, \quad \phi(L, t)=0, \quad l>0 .
$$

A second application of (15) is found in a solid state problem [7]. If the variables $i$ and $C$ are eliminated in equation (3) of [7], one obtains the ordinary differential equation

$$
\phi^{\prime \prime}+b \phi^{\prime}=2 \phi^{-1} \phi^{\prime 2}-g \phi^{2}
$$


with constant coefficients, which is to be solved subject to the initial conditions

$$
\phi(0)=1, \quad \phi^{\prime}(0)=0 .
$$

The solution of $(26)$ is

$$
\phi(t)=[u(t)-\beta]^{-1},
$$

the solution $u$ being

$$
u(t)=C_{1}+C_{2} \exp (-b t)+g t / b
$$

with $C_{1}$ and $C_{2}$ arbitrary constants. Imposing condition (11), one obtains

$$
u(0)=1+\beta, \quad l=-1,
$$

which also follows from (29) with $C_{1}=1$ and $C_{2}=\beta$. Imposing condition (13) on $u^{\prime}(t)$, one must have $u^{\prime}(0)=0$. Differentiation of (29) with $C_{1}$ and $C_{2}$ equal to 1 and $\beta$, respectively, shows that $u^{\prime}(0)=0$ is secured if $\beta=g / b^{2}$. The initial value problem (26) and (27) is thus solved.

\section{REFERENCES}

1. R. T. Herbst, The equivalence of linear and nonlinear differential equations, Proc. Amer. Math. Soc. 7 (1956), 95-97. MR 17, 848.

2. D. V. Widder, Series expansions of solutions of the heat equation in $n$ dimensions, Ann. Mat. Pura Appl. (4) 55 (1961), 389-409. MR 25 \#331.

3. C. Y. Lo, Series expansions of solutions of $U_{x x}+U_{y y}+\varepsilon^{2} U_{t t}=U_{t}$, SLAM J. Math. Anal. 3 (1972), 461-473.

4. E. W. Montroll, On nonlinear processes involving population growth and diffusion, J. Appl. Probability 4 (1967), 281-290. MR 36 \#3585.

5. - Lectures on nonlinear rate equations, especially those with quadratic nonlinearities, Lectures in Theoretical Physics 10A: Quantum theory and statistical physics, Gordon and Breach, New York, 1968, pp. 531-573. MR 38 \#4099a.

6. A. N. Tihonov and A. A. Samarskiī, Equations of mathematical physics, GITTL, Moscow, 1951; English transl., Pergamon, Oxford and Macmillan, New York, 1963. MR 16, 130; 29 \#2498.

7. A. C. Damask and G. J. Dienes, Kinetics of vacancy-interstitial annihilation. I. Impurity-interstitial trapping, Phys. Rev. 125 (1962), 444-446.

Department of Physics and Astronomy, Clemson University, Clemson, South Carolina 29631

Department of Physics, Old Dominion University, Norfolk, Virginia 23508 